\title{
SHARP BOUNDS FOR THE CONVEX COMBINATIONS OF ARITHMETIC, LOGARITHMIC AND GEOMETRIC MEANS IN TERMS OF HARMONIC MEAN
}

\author{
ZAI-YIN HE AND YU-MING CHU ${ }^{\dagger}$ \\ Date of Receiving : $\quad$ 11.05. 2014 \\ Date of Revision : $\quad$ 08. 06. 2014 \\ Date of Acceptance : 27.06 .2014
}

\begin{abstract}
In this paper, we find the greatest values $r_{1}$ and $r_{2}$, and the least values $s_{1}$ and $s_{2}$ in $(0,1 / 2)$ such that the double inequalities $H\left[r_{1} a+\left(1-r_{1}\right) b, r_{1} b+\left(1-r_{1}\right) a\right]<$ $\alpha A(a, b)+(1-\alpha) L(a, b)<H\left[s_{1} a+\left(1-s_{1}\right) b, s_{1} b+\left(1-s_{1}\right) a\right]$ and $H\left[r_{2} a+\left(1-r_{2}\right) b, r_{2} b+\left(1-r_{2}\right) a\right]<$ $\alpha A(a, b)+(1-\alpha) G(a, b)<H\left[s_{2} a+\left(1-s_{2}\right) b, s_{2} b+\left(1-s_{2}\right) a\right]$ hold for all $a, b>0$ with $a \neq b$ and any $\alpha \in(0,1)$, where $H(a, b), G(a, b), L(a, b)$ and $A(a, b)$ are the harmonic, geometric, logarithmic and arithmetic means of two positive numbers $a$ and $b$, respectively.
\end{abstract}

\section{Introduction}

For $a, b>0$ with $a \neq b$, the classical harmonic mean $H(a, b)$, geometric mean $G(a, b)$, arithmetic mean $A(a, b)$, logarithmic mean $L(a, b)$, identric mean $I(a, b)$ are defined by

$$
\begin{gathered}
H(a, b)=\frac{2 a b}{a+b}, G(a, b)=\sqrt{a b}, A(a, b)=\frac{a+b}{2}, \\
L(a, b)=\frac{a-b}{\log a-\log b}, I(a, b)=\frac{1}{e}\left(\frac{b^{b}}{a^{a}}\right)^{1 /(b-a)},
\end{gathered}
$$

respectively. It is well known that the inequalities

$$
H(a, b)<G(a, b)<L(a, b)<I(a, b)<A(a, b)
$$

hold for all $a, b>0$ with $a \neq b$.

2010 Mathematics Subject Classification. 26E60, 26D15.

Key words and phrases. harmonic mean, geometric mean, logarithmic mean, arithmetic mean. The authors would like to express their deep gratitude to the referee for giving many valuable suggestions. This research was supported by the Natural Science Foundation of China under Grants 11171307 and 61374086, and the Natural Science Foundation of Zhejiang Province under Grant LY13A010004.

Communicated by. Virender

${ }^{\dagger}$ Corresponding author. 